\title{
Primary and secondary structures of rRNA spacer regions in enterococci
}

\author{
Afaf Naïmi, Geneviève Beck and Christiane Branlant \\ Author for correspondence: Christiane Branlant. Tel: +33 38391 20 91. Fax: +33 383912093. \\ e-mail : cbranlant@legg.u-nancy.fr
}

Laboratoire d'Enzymologie et de Génie Génétique, URA CNRS 457, Université de Nancy I, Bld des Aiguillettes, BP 239-54506 Vandœuvre-Lès-Nancy Cedex, France

\begin{abstract}
The 16S-23S and 235-5S rRNA spacer DNA regions (spacer regions 1 and 2, respectively) from Enterococcus faecalis, Enterococcus faecium, Enterococcus hirae, Enterococcus durans and Enterococcus mundtii were amplified by PCR. Their nucleotide sequences were established and a secondary structure model showing the interaction between the two spacer regions was built. Whereas lactococci and Streptococcus sensu stricto are characterized by a single type of spacer region 1, the enterococci show a high degree of variability in this region; thus the spacer regions 1 with and without tRNA $A^{\text {Ala }}$ were characterized. However, as shown for lactococci and Streptococcus sensu stricto, the tRNA ${ }^{\text {Ala }}$ gene does not encode the 3'-terminal CCA trinucleotide. A putative antitermination signal is found downstream from the tRNA ${ }^{\text {Ala }}$ gene. Based on comparison with Lactococcus lactis and Streptococcus thermophilus, a doublestranded processing stem is proposed. In E. hirae, one of the three different types of spacer region 1 contains no tRNAAla, but displays a 107 nt insertion that forms a long stem-loop structure. A similar insertion (115 nt in length) was found in $E$. faecium and base compensatory mutations preserve the ability to form the long stem-loop structure. Such insertions may correspond to mobile intervening sequences, as found in the 235 rRNA coding sequences of some Gram-negative bacteria. The spacer regions 1 and 2 from the three subgroups of streptococci were compared, and except for the tRNAAla gene and the double-stranded processing sites, little similarity was found, which opens large possibilities for future development of DNA-based typing methods.
\end{abstract}

Keywords: $16 \mathrm{~S}-23 \mathrm{~S}$ rRNA spacer, 23S-5S rRNA spacer, tRNA ${ }^{\text {Ala }}$, enterococci

\section{INTRODUCTION}

The enterococci are increasingly recognized as common causes of infection that may become difficult to treat because of both inherent and acquired antibiotic resistance (Hall et al., 1992). Enterococci are characterized by their ability to grow at 10 and $45^{\circ} \mathrm{C}$, in $6.5 \% \mathrm{NaCl}$ at $\mathrm{pH} 9 \cdot 6$, and to hydrolyse esculin in the presence of $40 \%$ bile (Mundt, 1986). However, several of the recently described species do not conform to these phenotypic characteristics (Devriese et al., 1993). Hence, the development of rapid and reliable DNA-based methods for the identification of Enterococcus isolates is of medical importance.

\footnotetext{
Abbreviations: dsPS, double-stranded processing stem; LS1, large spacer region 1 ; SS1, small spacer region 1 .

The GenBank/EMBL/DDBJ accession numbers for the sequences reported in
} this paper are given in Table 1.
Based on 165 rRNA nucleotide sequence determination and nucleic acid hybridization, the previous Bergey's classification of Gram-positive bacteria (Hardie, 1986) has been revised, and enterococci, lactococci and Streptococcus sensu stricto were found to be three genetically distinct subgroups (Kilpper-Bälz et al., 1982; Ludwig et al., 1985; reviewed by Schleifer \& KilpperBälz, 1987). According to these nucleic acid analyses, the genus Enterococcus was also divided into 'species groups' (Williams et al., 1991). The 'faecium group' consists of the Enterococcus durans, Enterococcus faecium, Enterococcus hirae and Enterococcus mundtii species, the 'avium group' of the Enterococcus avium, Enterococcus maladoratus and Enterococcus pseudoavium species and the 'gallinarum group' of the species pair Enterococcus casseliflavus and Enterococcus gallinarum. Other species, such as Enterococcus faecalis, were found to form distinct lineages. In spite of this great progress in the classification of enterococci, only 
limited efforts have been made to develop DNA-based typing methods for their rapid identification. The main achievement was the design of two 20-mer oligonucleotides as 23S-rRNA-targeted probes for the identification of E. faecium and E. faecalis (Betzl et al., 1990, Beimfohr et al., 1993).

The 16S-23S spacer region (spacer region 1) from the bacterial rRNA genetic loci $(r r n)$ is an important tool for the development of DNA-based typing methods because it shows a significant degree of variation in length and sequence from one species to the other (Barry et al., 1991; van der Giessen et al., 1994; review by Gürtler \& Stanisich, 1996). In addition a given species may be characterized by a defined polymorphism of spacer region 1 and this may also be used for rapid characterization of bacterial species (Jensen et al., 1993; Whiley et al., 1995). The most direct and certainly the most rapid method of visualizing the polymorphic character of $r r n$ spacer regions is PCR amplification of these spacer regions (Jensen et al., 1993). In the case of enterococci, only the spacer region 1 of two species, $E$. hirae and E. faecalis were studied, and a polymorphism was found for both of them. Two types of spacer regions 1 differing by the presence or the absence of a tRNA ${ }^{\text {Ala }}$ gene were identified, by direct cloning of genomic DNA fragments in the case of E. hirae (Sechi \& Daneo-Moore, 1993), and by PCR amplification for E. faecalis (Hall, 1994). Whereas the absence of a tRNA Ala gene in spacer region 1 is accompanied by a size reduction in $E$. faecalis (Hall, 1994), this is not the case in E. hirae (Sechi \& Daneo-Moore, 1993). This polymorphism of the spacer region 1 in enterococci is in contrast with the observation of an identical spacer region 1 sequence (Nour et al., 1995) in the five or six rrn operons of Lactococcus lactis, Streptococcus thermophilus and Streptococcus salivarius (Pébay et al., 1992; Le Bourgeois et al., 1995). A similar number of $r r n$ operons was proposed for E. hirae
(Sechi \& Daneo-Moore, 1993). The question is whether the polymorphism observed for the six operons of $E$. hirae (Sechi \& Daneo-Moore, 1993) is a general feature of enterococci.

Based on detailed studies made on the rRNA maturation process of Escherichia coli (review by Apirion \& Miczak, 1993), both spacer region 1 and the 23S-5S rRNA spacer region (spacer region 2), play a crucial role in pre-rRNA maturation. Studies on Esch. coli showed that base-pair interactions formed by the leader region upstream of $16 \mathrm{~S}$ rRNA and spacer region 1 on the one hand, and by spacer regions 1 and 2 on the other hand, generate the two primary double-stranded processing sites (dsPS1 and dsPS2), that are cleaved by RNase III (Apirion \& Miczak, 1993). Although only a limited number of $r r n$ spacer regions have been characterized (reviewed by Gürtler \& Stanisich, 1996), the presence of long double-stranded regions that close the $16 \mathrm{~S}$ and $23 \mathrm{~S}$ rRNA sequences and contain the primary processing sites seem to be a general feature of bacterial prerRNAs, they are not always found in archaeobacteria (Potter et al., 1995). A model of the interaction between spacer regions 1 and 2 that is supported by results of $\mathrm{S} 1$ nuclease digestion was proposed for $L$. lactis (Chiaruttini \& Milet, 1993), and we recently proposed a model of interaction for the spacer regions 1 and 2 of $S$. thermophilus and S. salivarius (Nour et al., 1995). To establish a model of interaction between spacer regions 1 and 2 for the third genus of streptococci, namely Enterococcus, the nucleotide sequence of a spacer region 2 from enterococci had to be determined. Determination of an enterococcal spacer region 2 nucleotide sequence is also of interest for the study of $5 \mathrm{~S}$ rRNA maturation. Indeed, RNase E, which is involved in $5 \mathrm{~S} \mathrm{rRNA}$ maturation in Esch. coli, has a recognition site within spacer region 2 (5'-ACAGAAUUUG-3') (reviewed by Apirion \& Miczak, 1993).

Table 1. EMBL database accession numbers for the intergenic rRNA spacer regions established in this paper

\begin{tabular}{|c|c|c|c|}
\hline Strain & EMBL no. & Spacer region* & Length (bp) \\
\hline E. faecalis ATCC $19433^{\mathrm{T}}$ & X87186 & $1, \mathrm{tRNA}^{\mathrm{Ala}}$ & 328 \\
\hline E. faecalis ATCC $19433^{\mathrm{T}}$ & X81782 & 1 & 226 \\
\hline E. faecalis ATCC $19433^{\mathrm{T}}$ & X87183 & 2 & 96 \\
\hline E. durans ATCC $19432^{\mathrm{T}}$ & X87177 & $1, \mathrm{tRNA}^{\mathrm{Ala}}$ & 330 \\
\hline E. durans ATCC $19432^{\mathrm{T}}$ & X87178 & 1 & 277 \\
\hline E. durans ATCC $19432^{\mathrm{T}}$ & X87179 & 2 & 92 \\
\hline E. faecium ATCC $19434^{\mathrm{T}}$ & X87180 & 1 & 344 \\
\hline E. faecium ATCC $19434^{\mathrm{T}}$ & X87181 & 2 & 94 \\
\hline E. faecium LMA631 & X87190 & 1 & 344 \\
\hline E. faecium LMA631 & X87191 & 2 & 94 \\
\hline E. hirae ATCC $8043^{\mathrm{T}}$ & X87184 & 1 & 232 \\
\hline E. hirae ATCC $8043^{\mathrm{T}}$ & X87185 & 2 & 94 \\
\hline E. mundtii ATCC 582 & X87187 & $1, \mathrm{tRNA}^{\mathrm{Ala}}$ & 331 \\
\hline E. mundtii ATCC 582 & X87188 & 1 & 230 \\
\hline E. mundtii ATCC 582 & X87189 & 2 & 89 \\
\hline
\end{tabular}

*1, 16S-23S rRNA spacer DNA; 2, 23S-5S rRNA spacer DNA. 
Hence, determination of the nucleotide sequence of spacer regions 1 and 2 of a series of enterococci with a study of their polymorphism was expected to bring new insight on the structure and the evolution of the prerRNA spacer region in streptococci and to be a prerequisite for the future development of DNA-based methods for the identification of enterococci.

In this paper, we describe an exhaustive analysis of the spacer regions 1 and 2 from E. durans, E. faecium, E. hirae, E. mundtii and E. faecalis using PCR and direct sequence analysis of the amplification products. For each species, an RNA secondary structure model showing the interaction between the two spacer regions 1 and 2 was built. The results obtained are discussed in the light of the present knowledge on rRNA and tRNA processing. The possible utilization of the established sequences for the development of rapid DNA-based typing methods is also discussed.

\section{METHODS}

Bacterial strains and cultures. The enterococcal strains used in this study are listed in Table 1. All strains were stored at $-20^{\circ} \mathrm{C}$ in Brain-Heart Infusion (BHI) medium (Diagnostics Pasteur) in the presence of $15 \%$ (v/v) glycerol. Cultures were grown at $37^{\circ} \mathrm{C}$ in BHI medium for E. faecalis, E. durans, $E$. faecium ATCC $19434^{\mathrm{T}}$, E. hirae and E. mundtii and in lac/MRS (De Man et al., 1960) liquid medium for E. faecium LMA631. For E. mundtii ATCC 582, BHI medium was supplemented with $10 \%(\mathrm{v} / \mathrm{v})$ horse blood as recommended by the Pasteur Institute strain distribution service. Culture purity was checked on nutrient agar (10 g tryptone $\mathrm{l}^{-1}, 5 \mathrm{~g}$ yeast extract $\left.\mathrm{l}^{-1}, 5 \mathrm{~g} \mathrm{NaCl}^{-1}, 15 \mathrm{~g}_{\text {agar l}}{ }^{-1}\right)$. E. mundtii was spread on Colombia agar (Diagnostic Pasteur).

Isolation of DNA and PCR. Enterococcal genomic DNA was prepared from early stationary-phase cells according to Hill $e t$ al. (1990) with modifications to temperature and duration of incubations as previously described (Nour et al., 1995). PCR amplifications were performed as described by Nour et al. (1995). The DNA fragments encoding the 16S-23S rRNA spacer region (spacer region 1) were amplified using primer combination 1: primer 514, 5'-TGGATCACCTCCTTTCTA-3' (sequence of the $18 \mathrm{nt}$ at the $3^{\prime}$-end of Streptococcus pneumoniae 16S rRNA; Bacot \& Reeves, 1991) and primer 554, 5'-ACCGTGTACGCTTAGTCGCTTAA-3', which is complementary to a highly conserved $23 \mathrm{~S}$ rRNA sequence (positions 1-25 of the Esch. coli 23S rRNA; Branlant et al., 1981). The DNA fragments encoding the 23S-5S rRNA spacer region (spacer region 2) were amplified using primer combination 2: primer 601, $5^{\prime}$-CTTAACTTCTGTGTTCGGCATG-3', which is complementary to nucleotide positions 35-53 of the L. lactis 5S rRNA (Chiaruttini \& Milet, 1993) and primer $667, \quad 5^{\prime}$-CTAATAGGTCGAGGACTTGACCAA-3' (sequence of the S. thermophilus $23 \mathrm{~S}$ rRNA between positions 2876 and 2900; Ludwig et al., 1992).

PCR product purification and sequencing. PCR products were recovered from the incubation mixture by phenol extraction, followed by phenol/chloroform/isoamyl alcohol $(25: 24: 1$, by vol.) and chloroform/isoamyl alcohol $(24: 1, \mathrm{v} / \mathrm{v})$ extractions. DNA was precipitated at $-20^{\circ} \mathrm{C}$, with $2.5 \mathrm{M}$ ammonium acetate and 1 vol. ethanol. When necessary, amplified DNA fragments were purified by electrophoresis on $6 \%(\mathrm{w} / \mathrm{v})$ polyacrylamide gel made up in TBE buffer (Sambrook et al.,

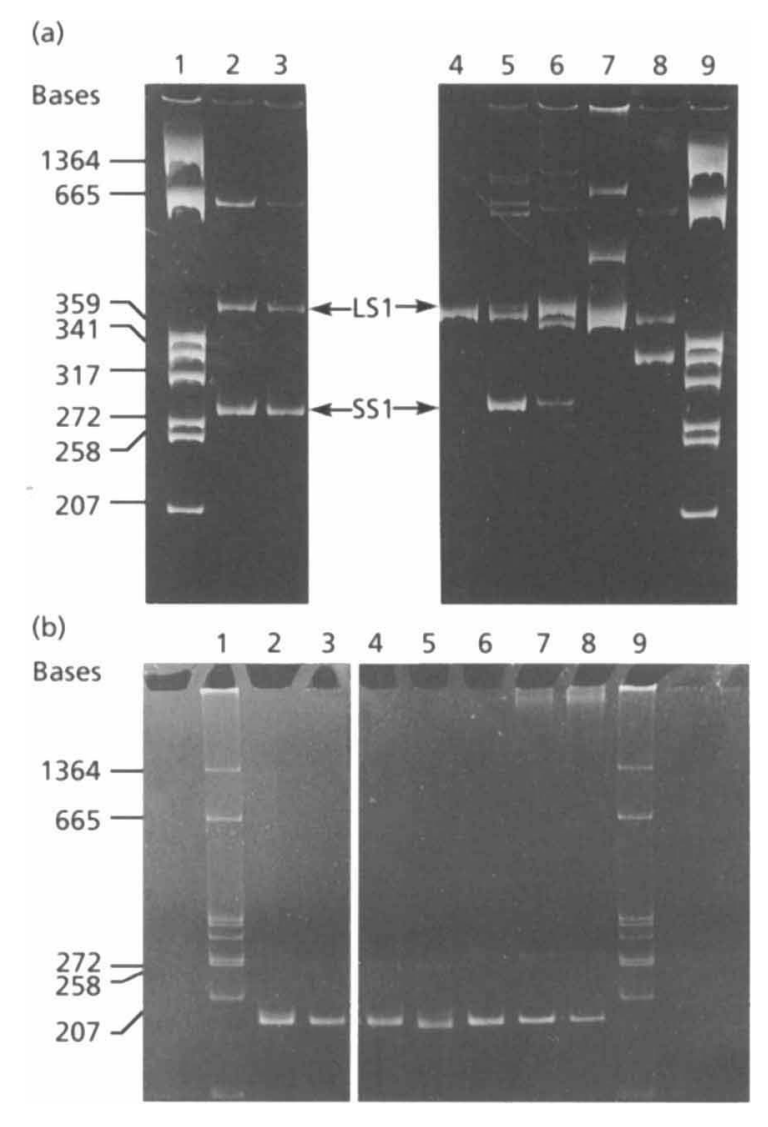

Fig. 1. $P C R$ analysis of the enterococcal rRNA spacer regions 1 and 2. Genomic DNA was prepared as described in Methods and amplified (a) with primer combination 1 and (b) with primer combination 2 . The amplified DNA fragments were fractionated by electrophoresis on a $6 \%$ polyacrylamide gel and visualized by ethidium bromide fluorescence. Lanes: $2, E$. faecalis ATCC 5430; 3, E. faecalis ATCC 19433; 4, E. faecium LMA631; 5, E. mundtii ATCC 582; 6, E. hirae ATCC 8043; 7, E. faecium ATCC $19434 ; 8$, E. durans ATCC 19432; 1 and 9 , pBR322 cleaved by SauBAl, the sizes of the fragments are indicated on the left. Abbreviations: SS1, small spacer region 1; LS1, large spacer region 1.

1989). Gels were stained with ethidium bromide and DNA was visualized by UV fluorescence. Elution was according to the methods of Sambrook et al. (1989). The complete sequences of the amplified fragments were determined on both strands as described by Nour et al. (1995). Oligonucleotides $514,554,601$ and 667 were used as primers for sequence analysis. The annealing reaction was for $3 \mathrm{~min}$ at $94^{\circ} \mathrm{C}$ in the annealing buffer $(50 \mathrm{mM} \mathrm{NaCl}, 10 \mathrm{mM} \mathrm{MgCl}, 40 \mathrm{mM}$ Tris/ $\mathrm{HCl}, \mathrm{pH} 7 \cdot 5$ ). The annealing mixture was immediately cooled on ice for $10 \mathrm{~min}$ and used for elongation in the presence of T7 DNA polymerase (Pharmacia) (Tabor \& Richardson, 1987).

Computer sequence analysis. The computer program CLUSTAL w (Thompson et al., 1994) was used for sequence alignment and the Alscript program (Borton, 1993) for representation of sequence similarities. The Mfold program of the Genetics Computing Group (GCG, University of Wisconsin, USA) software version 8 was used for prediction of RNA secondary structure. 

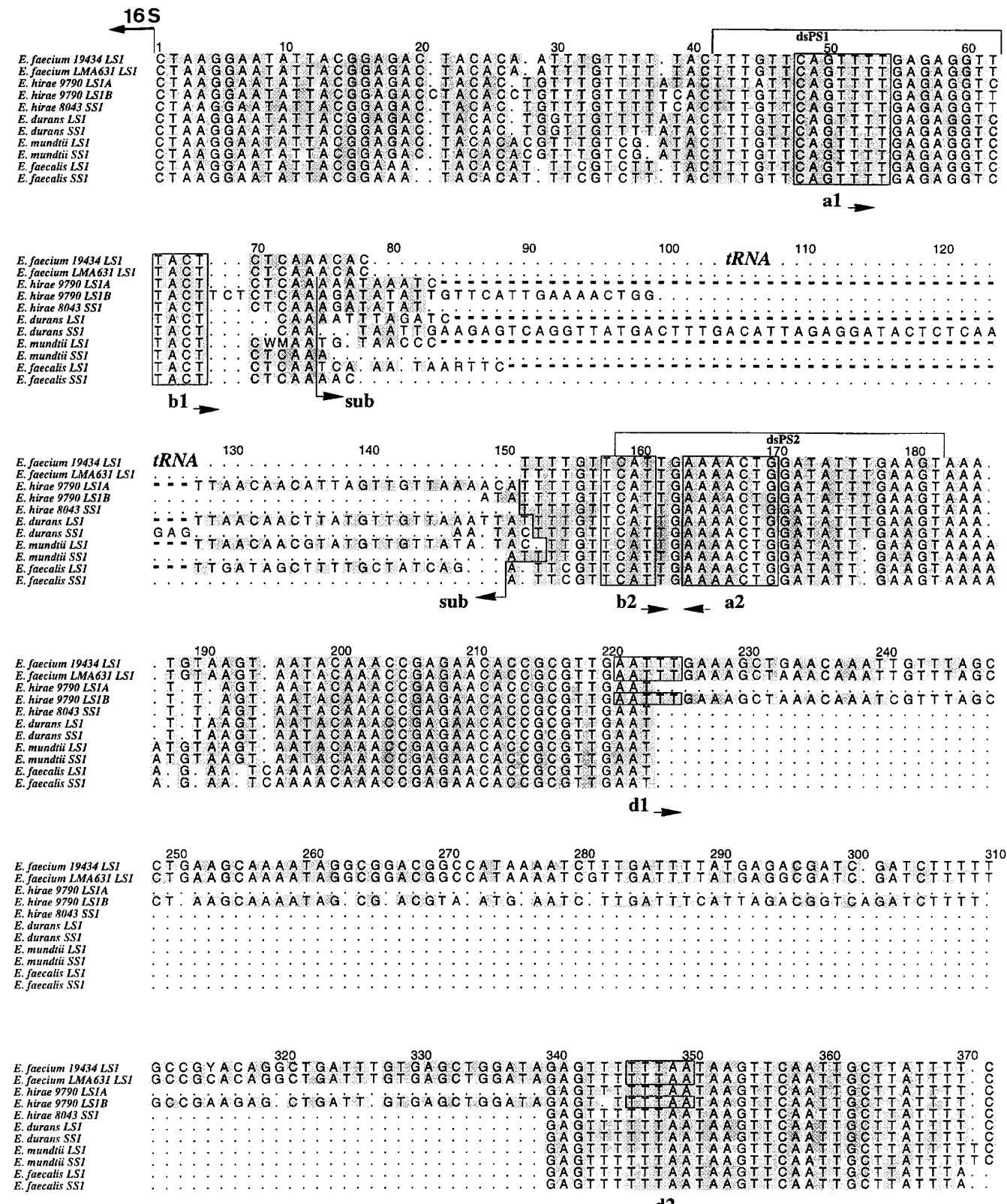

$\sim \mathrm{d} 2$

$\stackrel{23 S}{\longrightarrow}$
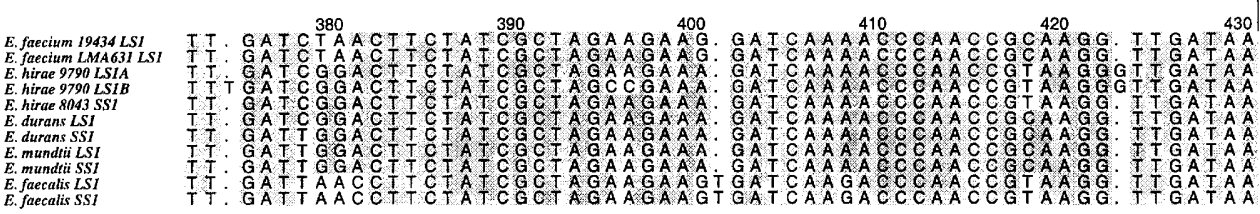

Fig. 2. Nucleotide sequence comparison of enterococcal spacer regions 1 . All the enterococcal LS1 and SS1 fragments sequenced in this study were aligned with the CLUSTAL W program (Thompson et al., 1994). The LS1 spacer regions with (LS1A) and without (LS1B) tRNAAla, previously sequenced for E. hirae ATCC 9790 (Sechi \& Daneo-Moore, 1993), are included in the comparison. The $16 \mathrm{~S}$ and $23 \mathrm{~S}$ rRNA sequences at the extremities of the amplified fragments were excluded from the comparison. Similarities were represented by the Alscript program (Borton, 1993). When at a given position the same nucleotide is found in at least three of the aligned sequences, it is shaded in all the sequences where it is present. Sequence heterogeneities are indicated by the usual nomenclature ( $M$ for $A$ or $C, R$ for $A$ or $G, Y$ for $C$ or $T$, and $\mathrm{W}$ for $\mathrm{A}$ or T). The borders of the region that differs in the LS1 and SS1 fragments are denoted by a vertical line marked by sub on the left and by a broken line marked by sub on the right. The tRNA sequence is represented by a horizontal dashed line. The sequences involved in the putative dsPS1 and dsPS2 stems are overlined. The pairs of palindromic sequences $\mathrm{a} 1 / \mathrm{a} 2$ and the two pairs of directly inverted sequences (b1/b2 and d1/d2) are boxed. 


\section{RESULTS}

\section{Length polymorphism of the enterococcal spacer region 1}

The type strain was selected for E. faecalis, E. durans, E. faecium and E. hirae, and the ATCC strain 582 was selected for E. mundtii (Table 1). For E. faecalis and E. faecium, a second strain was introduced in the collection for evaluation of the intraspecific degree of conservation. Primer combinations 1 and 2 (Methods) were used for PCR amplification of the $r r n$ spacer regions 1 and 2, respectively. For all the species studied, primer combination 2 generated a unique amplification product of about 100 bp (Fig. 1b). A larger number of fragments were amplified with primer combination 1 , and their lengths varied from one species to the other (Fig. 1a). One or two amplification products of about $300 \mathrm{bp}$ were obtained for all the strains (LS1, for large spacer region 1). A smaller fragment of about $220 \mathrm{bp}$ was found for $E$. faecalis, E. mundtii and E. hirae and a fragment with an intermediate size (about $270 \mathrm{bp}$ ) for E. durans. These fragments were denoted SS1, for small spacer region 1. Finally, very large fragments varying in number, size and intensity were obtained for most of the species, except E. faecium LMA631. It is not clear whether these large fragments $(500-1000 \mathrm{bp})$, corresponded to amplified $\mathrm{rDNA}$, since the yield of some of them was significantly lower than those of the amplified fragments LS1 and SS1. Similar observations were made by Hall (1994) for E. faecalis ATCC 19433. Thus, only the LS1 and SS1 fragments were sequenced. For E. hirae, the two LS1 fragments were too close to be resolved. They were not sequenced since according to their sizes, they corresponded to the two spacer regions 1 that were previously cloned and sequenced for $E$. hirae ATCC 9790 (Sechi \& Daneo-Moore, 1993). E. mundtii also had a pair of LS1 fragments, one of which was largely prominent and this was sequenced. DNA fragments amplified with primer combination 2 were also sequenced; these are denoted S2 (for spacer region 2).

\section{Nucleotide sequences of enterococcal spacer regions 1 and 2}

None of the SS1 fragments contained a tRNA gene. In contrast, all LS1 fragments, except for E. faecium, contained a tRNA ${ }^{\text {Ala }}$ gene. The nucleotide sequences for the SS1 and LS1 fragments are aligned in Fig. 2, together with the nucleotide sequences of the two LS1s sequenced for E. hirae ATCC 9790 (Sechi \& Daneo-Moore, 1993). Sequences from DNA fragments amplified with primer combination 2 are aligned in Fig. 3.

For both spacer regions, no sequence differences were detected between E. faecalis ATCC 19433 and ATCC 5430 , and the sequences that we established for the SS1 and LS1 fragments of these two strains are in perfect agreement with those published by Hall (1994) for ATCC 19433. This author also studied two clinical isolates (617 and 805). In the LS1 fragment, Hall (1994) found four single-base differences between strain ATCC 19433 and the two clinical isolates. At one of these four positions (position 85 in Fig. 2), an A/G heterogeneity was suspected in E. faecalis ATCC 19433, and our sequencing data confirmed the presence of this heterogeneity. We also detected two micro-heterogeneities in the LS1 fragment of E. mundtii ATCC 582 (positions 71 and 72 in Fig. 2). The LS1 fragments of E. faecium ATCC 19434 and LMA631 differed at four positions, at one of these positions (315 in Fig. 2), a $C$ residue was read on the sequencing gel for E. faecium LMA631, whereas both $C$ and $U$ residues were detected at this position for E. faecium ATCC 19434. The three other differences (positions 233, 281 and 295 in Fig. 2) are single-base substitutions between the two strains. Micro-heterogeneity seems to be a specific feature of the LS1. No micro-heterogeneity was detected in the SS1 or S2 fragments. We should point out that detection of two different nucleotides at a given position was not the result of sequencing or PCR artefacts, since the two complementary nucleotides were also detected upon sequencing the complementary DNA strand, and the

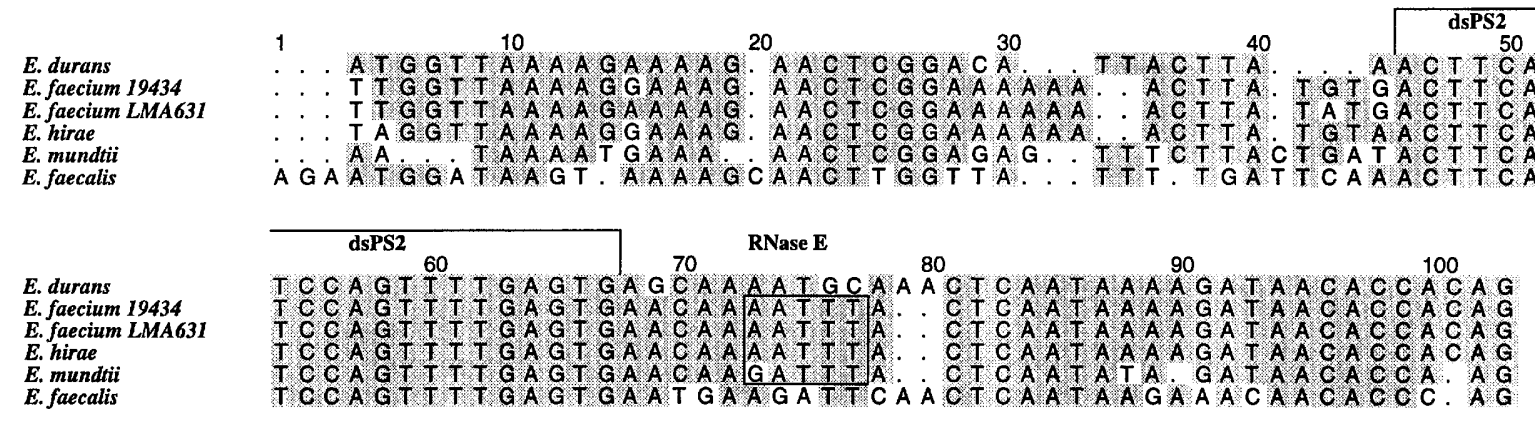

Fig. 3. Nucleotide sequence comparison of enterococcal spacer region 2. The sequences established from the DNA fragments amplified with the primer combination 2 were aligned with the CLUSTAL $W$ program. Similarities were represented with the Alscript program using the same rules as in Fig. 2. The nucleotide sequences involved in formation of the putative dsPS2 stem are overlined and the putative RNAase E recognition sites in E. faecium, E. hirae and E. mundtii are boxed. 
(a)

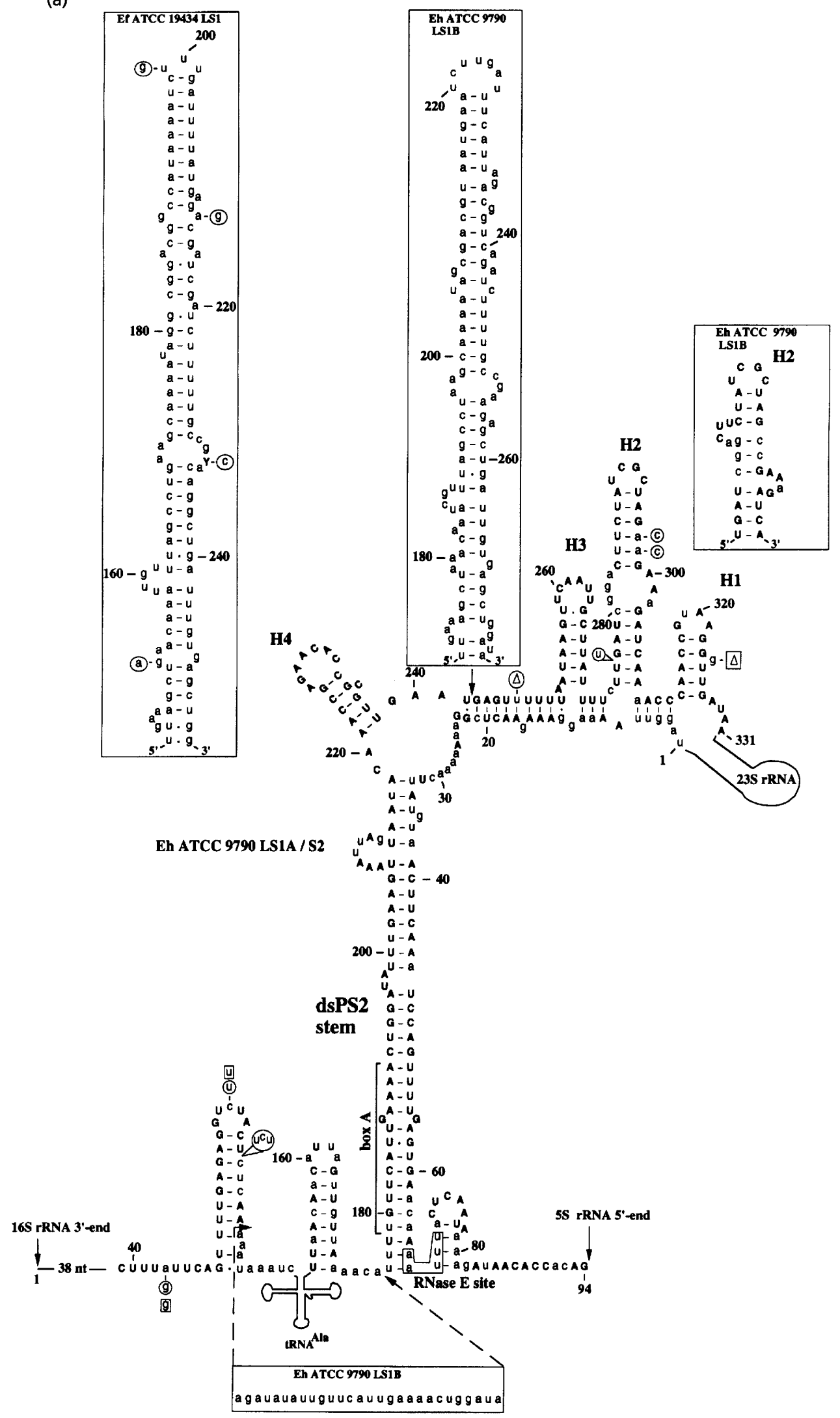

Fig. 4. For legend see facing page. 
(b)

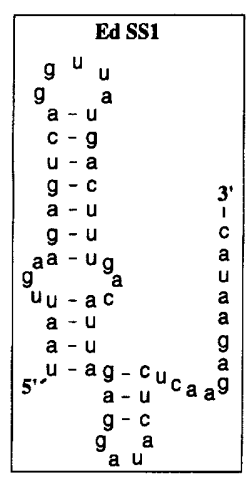

\section{Em LS1 / S2}
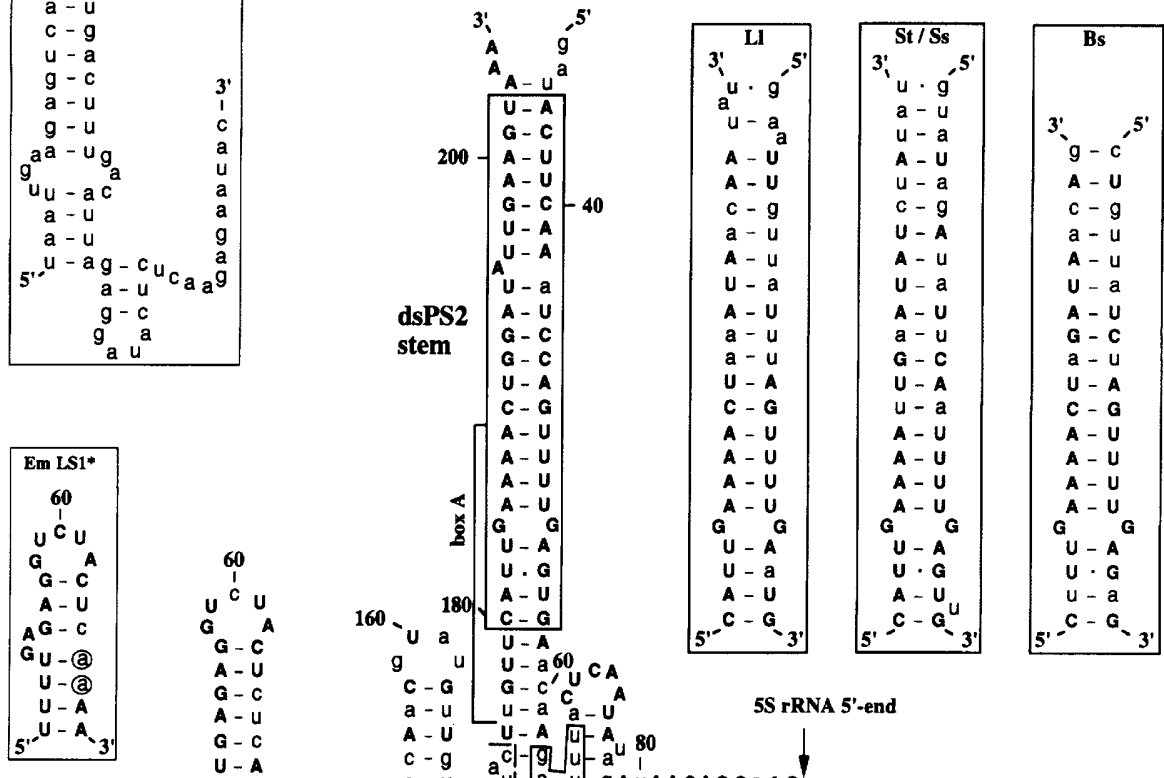

$c-g$ acj-g] $u-a^{u}{ }^{80}$

A - U ul-a u.gauAaCACCaA

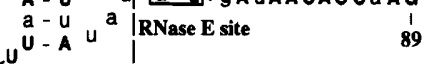

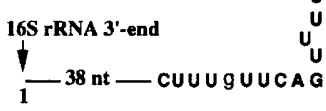

$\mathbf{G}-\mathrm{C}$
$\mathbf{U}-\mathbf{A}$

$-\frac{a}{4}$

$\cdot \log _{\mathrm{u}}$

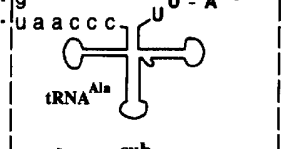

Fig. 4. Scheme for the secondary structure of the enterococcal pre-rRNA region between the dsPS1 stem and $5 S$ rRNA. A secondary structure model was established for each enterococcal species studied on the basis of the structure proposed for the corresponding pre-rRNA region of L. lactis (Chiaruttini \& Milet, 1993) and S. thermophilus (Nour et al., 1995) and by use of the GCG program Mfold. (a) Interaction between the LS1A spacer region of E. hirae ATCC 9790 and S2 spacer region of $E$. hirae ATCC 8043. Nucleotides are numbered starting from position 1 of each spacer region. Capital letters represent nucleotides conserved in the five enterococcal species studied. Extremities of $16 S$ and $5 S$ rRNAs are indicated by vertical arrows. The $23 \mathrm{~S}$ rRNA and the tRNA ${ }^{\mathrm{Ala}}$ are schematically represented. The putative $\mathrm{A}$ box of the antitermination signal and the putative RNase E site are indicated. All the differences found in the LS1B spacer region without tRNA from the same $E$. hirae strain are indicated: the $28 \mathrm{nt}$ sequence in place of the tRNA and bordering sequences, the $107 \mathrm{nt}$ insert, the rearrangement of stem-loop $\mathrm{H} 2$ and the single-base deletions $(\Delta)$ and mutations (circled nucleotides). The single-base deletions and mutations in the SS1 spacer region from $E$. hirae ATCC 8043 are indicated by $\Delta$ and nucleotides in squares, respectively. The $115 \mathrm{nt}$ insert from $E$. faecium ATCC 19434 is shown on the left, differences in the LMA631 strain are represented by circled nucleotides. (b) Part of the LS1/spacer region 2 interaction of $E$. mundtii including the dsPS2 stem. Nucleotides conserved in all the sequences established are indicated by capital letters. The effects of sequence heterogeneity on the stem-loop structure upstream of the tRNA ${ }^{\text {Ala }}$ is shown in the lower inset (LS1*) on the left (the two variant nucleotides are circled). The putative $A$ box of the antitermination signal and the putative RNase $E$ site are indicated as in (a). The extremities of the segment substituted by a $4 \mathrm{nt}$ sequence in the SS1 spacer region are indicated by arrows marked with sub, for substitution. The two stem-loop structures of the segment that replace the tRNA Ala and bordering sequence in the $E$. durans SS1 spacer region are shown in the upper inset on the left. The nucleotide sequences of the dsPS2 stems of L. lactis (LI; Chiaruttini \& Milet, 1993), S. thermophilus, S. salivarius (St/SS; Nour et al., 1995) and B. subtilis (Bs; Ogasawara et al., 1983) are shown in the three insets on the right, respectively. Nucleotides identical to those in enterococci are indicated by capital letters.

same relative levels of the two alternative nucleotides were reproducibly observed on independently amplified DNA fragments. The presence of sequence heterogeneities in the LS1 fragment indicates that at least two $r r n$ operons have a long version of the spacer region 1. As a final comment on strain sequence differences, we should point out that we found two differences between the spacer regions 2 of E. faecium ATCC 19434 and E. faecium LMA631 (positions 15 and 43 in Fig. 3).
Comparison of the SS1 and LS1 fragments for each species (Fig. 2) shows that they differ by the substitution of the tRNA ${ }^{\mathrm{Ala}}$ gene and bordering sequences in LS1 with a short nucleotide sequence in SS1. Whereas 3, 4 and 8 bp sequences replaced the tRNA ${ }^{\text {Ala }}$ gene in the $E$. faecalis, E. mundtii and E. hirae ATCC 8043 SS1 fragment, respectively, a longer sequence was found for E. durans (55 bp) (Fig. 2) and this explains the greater length of the E. durans SS1 fragment. The unexpected 
length of the E. faecium ATCC 19434 and LMA631 and E. hirae ATCC 9790 spacer regions 1 without tRNA ${ }^{\text {Ala }}$ gene is explained by the presence of a $115 \mathrm{bp}$ insertion in the E. faecium ATCC 19434 and LMA631 LS1 fragments without tRNA and a $107 \mathrm{bp}$ insertion in the E. hirae ATCC 9790 LS1 fragment without tRNA, as compared to the SS1 fragments without tRNA in other species. These insertions are located at the same position in the two species (Fig. 2). Interestingly, the four nucleotide sequence differences between the LS1 fragments of the two E. faecium strains studied are located in the $115 \mathrm{bp}$ insertion. The length, the position and also the sequence of the insert are highly conserved from E. hirae to $E$. faecium (Fig. 2). The situation for E. hirae is highly complex : it contains (i) $r r n$ operons without the $107 \mathrm{bp}$ insert, (ii) $r r n$ operons without the tRNA ${ }^{\text {Ala }}$ gene and with the $107 \mathrm{bp}$ insert, and (iii) $r r m$ operons without the 107 bp insert but with a tRNA ${ }^{\mathrm{Ala}}$ gene. As two highmolecular-mass DNA fragments were amplified in high yield for E. faecium ATCC 19434 (Fig. 1a), they may correspond to spacer regions 1 with a tRNA ${ }^{\text {Ala }}$ gene. However, no large fragment was detected for E. faecium LMA631, which suggests that this strain contains no spacer region 1 with a tRNA gene.

In addition to the $t R \mathrm{RA}^{\mathrm{Ala}}$ gene, an 8-12 nt sequence on the $5^{\prime}$-side of the tRNA and a 20-25 nt sequence on the 3 -side of the tRNA, are absent in spacer regions 1 without the tRNA ${ }^{\mathrm{Ala}}$ gene (Fig. 2). At the RNA level, the tRNA 3' bordering sequence forms a stem-loop structure (Fig. 4). For each of the species studied, except for the substitution of the tRNA gene and bordering sequences, the short and long versions of the spacer region 1 had the same sequence. The only exception was E. hirae, but the observed differences may be due to the fact that the SS1 is from E. hirae ATCC 8043 and the LS1s are from E. hirae ATCC 9790.

In all the species studied, the tRNA ${ }^{\text {Ala }}$ gene insertion was bracketed by a pair of directly inverted sequences $\left(5^{\prime}\right.$-TACT-3' $/ 5^{\prime}$-TCAT-3' ${ }^{\prime}$ denoted b1/b2 in Fig. 2$)$ and a pair of palindromic sequences $\left(5^{\prime}\right.$-CAGTTTT $-3^{\prime} / 5^{\prime}$ AAAACTG-3', denoted $\mathrm{a} 1 / \mathrm{a} 2$ in Fig. 2). Interestingly, partial mirrors of the $\mathrm{a} 1 / \mathrm{a} 2$ palindromic sequences were found at the junctions between the constant regions and the substitution area (Fig. 2). Finally, in spite of mutations (E. durans) or heterogeneity (E. mundtii), a conserved pentanucleotide sequence, $5^{\prime}$-CTCAA- $3^{\prime}$, was always found immediately upstream of the substitution area (Fig. 2). Some of these peculiar features may be related to the insertion/deletion events that generated the two kinds of spacer region 1 . Two directly inverted sequences $\left(5^{\prime}\right.$-AATTT- $3^{\prime} / 5^{\prime}$-TTTAA- $3^{\prime}$, denoted $\mathrm{d} 1 / \mathrm{d} 2$ in Fig. 2) also bracketed the $115 \mathrm{bp}$ insertion in $E$. faecium and the $107 \mathrm{bp}$ insertion in E. hirae.

The percentage similarities between the spacer regions 1 of the five species studied were calculated taking into consideration only the segments that were common to the LS1 and SS1 spacer regions. As the various spacer regions 1 sequenced for E. hirae differed by point mutations, each of them was compared individually to spacer regions 1 of other species (Table 2). In agreement with previous results on $16 \mathrm{~S}$ rRNA (Williams et al., 1991), the highest levels of similarity were observed for E. durans and E. hirae (92-96.9\%), E. durans and E. faecium $(96.3 \%$ ) and E. faecium and E. hirae (from 90 to $96 \%$ ), the lowest for $E$. faecalis compared with the four other species $(82.4-91.9 \%$ similarity). The degree of nucleotide sequence variation for spacer region 2 was higher than that for spacer region 1 (Table 2). However, as for spacer region 1, the lowest scores of similarities were found when E. faecalis was compared to the species from the 'faecium group' (52.8-67\% similarity), which is in accord with the idea that E. faecalis constitute a different phylogenetic branch.

\section{Secondary structure of spacer regions 1 and 2}

Using the established sequences, we built secondary structure models showing the interaction between spacer regions 1 and 2. The secondary structure models that we built contain a putative $23 \mathrm{~S}$ rRNA double-stranded processing site (dsPS2) and, when present, the tRNA ${ }^{\text {Ala }}$ (Fig. 4). The nucleotide sequence of the stem containing the putative dsPS2 site is highly conserved in the five enterococcal species (Figs 2, 3 and 4) and shows a strong sequence conservation when compared to the L. lactis (Chiaruttini \& Milet, 1993), S. thermophilus and S. salivarius (Nour et al., 1995) dsPS2 stem (Fig. 4b). The sequences that join the extremity of the 23S rRNA to the dsPS2 stem can be folded in a similar way in the five enterococci. Four lateral stem-loop structures denoted $\mathrm{H} 1-\mathrm{H} 4$ can be formed and their nucleotide sequence is highly conserved (Fig. 4a).

None of the sequence variations that we detected between two isolates of a given species or two operons of a given isolate disturb the proposed secondary structure. For instance, the three differences detected between the E. hirae ATCC 8043 SS1 and the E. hirae ATCC 9790 LS1 fragments concern single-stranded nucleotides (Fig. 4a, squared nucleotides). A larger number of differences are found between the two large spacer regions 1 (LS1A and LS1B) of E. hirae ATCC 9790; they also do not disturb RNA secondary structure (Fig. 4a, circled nucleotides). For instance, two successive A residues in the stem-loop structure $\mathrm{H} 2$ are mutated into $\mathrm{C}$ residues in the E. hirae LS1B spacer region with the $107 \mathrm{nt}$ insert, but the possibility of forming a stem-loop structure is conserved (Fig. 4a, inset LS1B). Two heterogeneities in the E. mundtii LS1 fragment are located in the stem-loop structure that precedes the tRNA ${ }^{\mathrm{Ala}}$, and stem-loop structures with similar stabilities can be formed with the two alternative sequences (Fig. 4b, inset LS1* circled nucleotides). The spacer regions 2 (S2) from the two E. faecium strains ATCC 19434 and LMA631, differ at two positions (Fig. $3)$. One mutation concerns a bulged nucleotide, the other one results in the replacement of a UG pair by a UA pair.

Antitermination signals were proposed to contribute to transcription efficiency of the rrn operons (reviewed by Berg et al., 1989). They consist of a B box that forms a 
Table 2. Similarities of the rRNA spacer regions 1 (boxed) and rRNA spacer regions 2 (not boxed) of enterococci

For the spacer region 2, the comparison was made on the entire spacer region. For spacer region 1, the substituted region (sub in Fig. 2), the $115 \mathrm{nt}$ insertion of the LS1 spacer region from E. faecium ATCC 19434 and the $107 \mathrm{nt}$ insertion of the LS1B spacer region from E. hirae ATCC 9790 (Fig. 2) were omitted from the comparison. For all species except E. hirae, the SS1 and LS1 sequences taken into consideration for the comparison were identical. Hence, for each comparison a single value is given for both the SS1 and LS1 sequences. However, for E. hirae, each SS1, LS1A and LS1B sequence was compared individually.

\begin{tabular}{|c|c|c|c|c|c|}
\hline Species & 1 & 2 & 3 & 4 & 5 \\
\hline \multicolumn{6}{|l|}{ 1. E. birae } \\
\hline SS1 & & $96 \cdot 9$ & $96 \cdot 0$ & $91 \cdot 5$ & $88 \cdot 7$ \\
\hline LS1A & & $96 \cdot 0$ & 92.9 & $90 \cdot 2$ & $86 \cdot 5$ \\
\hline LS1B & & $92 \cdot 0$ & $90 \cdot 2$ & $88 \cdot 4$ & $82 \cdot 4$ \\
\hline 2. E. durans & $86 \cdot 6$ & & $96 \cdot 3$ & $93 \cdot 8$ & $84 \cdot 8$ \\
\hline 3. E. faecium & $97 \cdot 8$ & $87 \cdot 7$ & & $92 \cdot 9$ & $91 \cdot 5$ \\
\hline 4. E. mundtii & $73 \cdot 0$ & $69 \cdot 0$ & $73 \cdot 0$ & & $91 \cdot 9$ \\
\hline 5. E. faecalis & $59 \cdot 1$ & $67 \cdot 0$ & $64 \cdot 8$ & $52 \cdot 8$ & \\
\hline
\end{tabular}

stem-loop structure, followed by an A box. Putative antitermination signals were detected in the spacer regions 1 of various Gram-positive bacteria, namely Bacillus subtilis (Ogasawara et al., 1983), Streptomyces ambofaciens (Pernodet et al., 1989), L. lactis (Chiaruttini \& Milet, 1993), and mycobacteria (Harasawa et al., 1992). The A box proposed for the spacer region 1 of $B$. subtilis has the sequence $5^{\prime}$-TGTTCTTTGAAA-3' and it overlaps the dsPS2 stem (Berg et al., 1989). A sequence differing at only one position $5^{\prime}$-TGTTCATTGAAA-3' is found at a similar position in all enterococcal spacer regions 1 studied (Fig. 4). In the LS1 spacer region, the stem-loop structure on the $3^{\prime}$-side of the tRNA ${ }^{\text {Ala }}$ is located at a correct distance to behave as a $\mathrm{B}$ box. The presence of a strong antitermination signal upstream of the $\mathrm{RNA}^{\mathrm{Ala}}$ gene may be required to overcome pauses of the RNA polymerase at the highly $\mathrm{G}+\mathrm{C}$-rich helical structures of the tRNA ${ }^{\mathrm{Ala}}$.

A sequence, $5^{\prime}$-ACAAAAUUUA-3', very similar to the Esch. coli RNase $\mathrm{E}$ recognition site $\left(5^{\prime}\right.$ ACAGAAUUUG-3' (Apirion \& Miczak, 1993) is found upstream of the E. faecium, E. hirae and E. mundtii $5 \mathrm{~S}$ rRNA gene (Figs 3 and 4). Surprisingly, no counterparts were found in the E. durans and E. faecalis spacer regions 2 (Fig. 3).

The 115 and the $107 \mathrm{bp}$ insertions that are found in the E. faecium and E. hirae LS1, respectively, form long stem-loop structures (Fig. 4a). The inserts of the two species are located at the same position within spacer region 1 in an internal loop. They show $76.6 \%$ similarity and have mutations that compensate each other to preserve helix formation (Fig. 4a). Since the four differences between the two E. faecium strains studied are located in internal loops or the terminal loop, there seems to be a strong selection pressure to preserve the helical structure of the insert.

The spacer region 1 with a tRNA ${ }^{\mathrm{Ala}}$ from $E$. durans and E. faecalis, L. lactis, S. thermophilus and S. pneumoniae were compared (Fig. 5a). The most conserved region is the tRNA gene. The two segments involved in the putative dsPS1 and dsPS2 stems are also highly conserved. Except for these segments and the 5 -terminus, the similarity observed was at the lower limit of significance. The enterococcal LS1 spacer region is larger than the spacer regions 1 of L. lactis and Streptococcus sensu stricto. The E. durans and E. faecalis spacer region 2 were also compared to the spacer region 2 of $L$. lactis and $S$. thermophilus (Fig. 5b). The degree of similarity is very poor, only a part of the sequence involved in the putative dsPS2 stem is conserved.

\section{DISCUSSION}

Based on the nucleotide sequence determination of the spacer regions 1 and 2, we propose a general secondary structure model showing the interaction of these two spacer regions in enterococci. This model is supported by the observation that sequence differences between species, strains or operons either compensate each other to preserve RNA secondary structure or do not affect RNA secondary structure. Our study reveals a high degree of plasticity of the enterococcal spacer region 1 : most of the enterococci studied had long and short versions of the spacer region 1 that differ by the absence or the presence of a tRNA ${ }^{\text {Ala }}$ gene. Among streptococci, this feature seems to be unique to the Enterococcus genus. Indeed, spacer regions 1 without tRNA ${ }^{\mathrm{Ala}}$ were found neither in L. lactis (Chiaruttini \& Milet, 1993) nor in species from the Streptococcus sensu stricto genus (Bacot \& Reeves, 1991; Nour et al., 1995; Whiley et al., 1995). The co-existence of spacer regions 1 with and without tRNA was already observed for $B$. subtilis (Loughney et al., 1982). However, in this case two tRNA genes, namely tRNA ${ }^{\mathrm{Ala}}$ and $\mathrm{tRNA}^{\mathrm{Ile}}$, are present in the long version of the spacer region 1 . According to the results obtained for E. faecium LMA631, some $E$. faecium strains may contain no tRNA gene in the spacer region 1 . A complete absence of tRNA gene in all spacer regions 1 of a given species is not a frequent situation, although it has been described for mycobacteria (Ji et al., 1994; van der Giessen et al., 1994).

The enterococcal LS1 has the characteristic feature found for the spacer region 1 of other streptococci: a unique tRNA ${ }^{\text {Ala }}$ gene that does not encode the 3 'terminal CCA sequence. Until now, such a feature had been found only in streptococci (Bacot \& Reeves, 1991; Chiaruttini \& Milet, 1993; Nour et al., 1995), lactobacilli (Pittet \& Hottinger, 1989) and some archaeobacteria (reviewed by Bacot \& Reeves, 1991). In Grampositive bacteria, post-transcriptional addition of the 3 'terminal CCA of tRNA has been reported previously: in a $B$. subtilis tRNA gene cluster, three out of 21 tRNA 
(a)
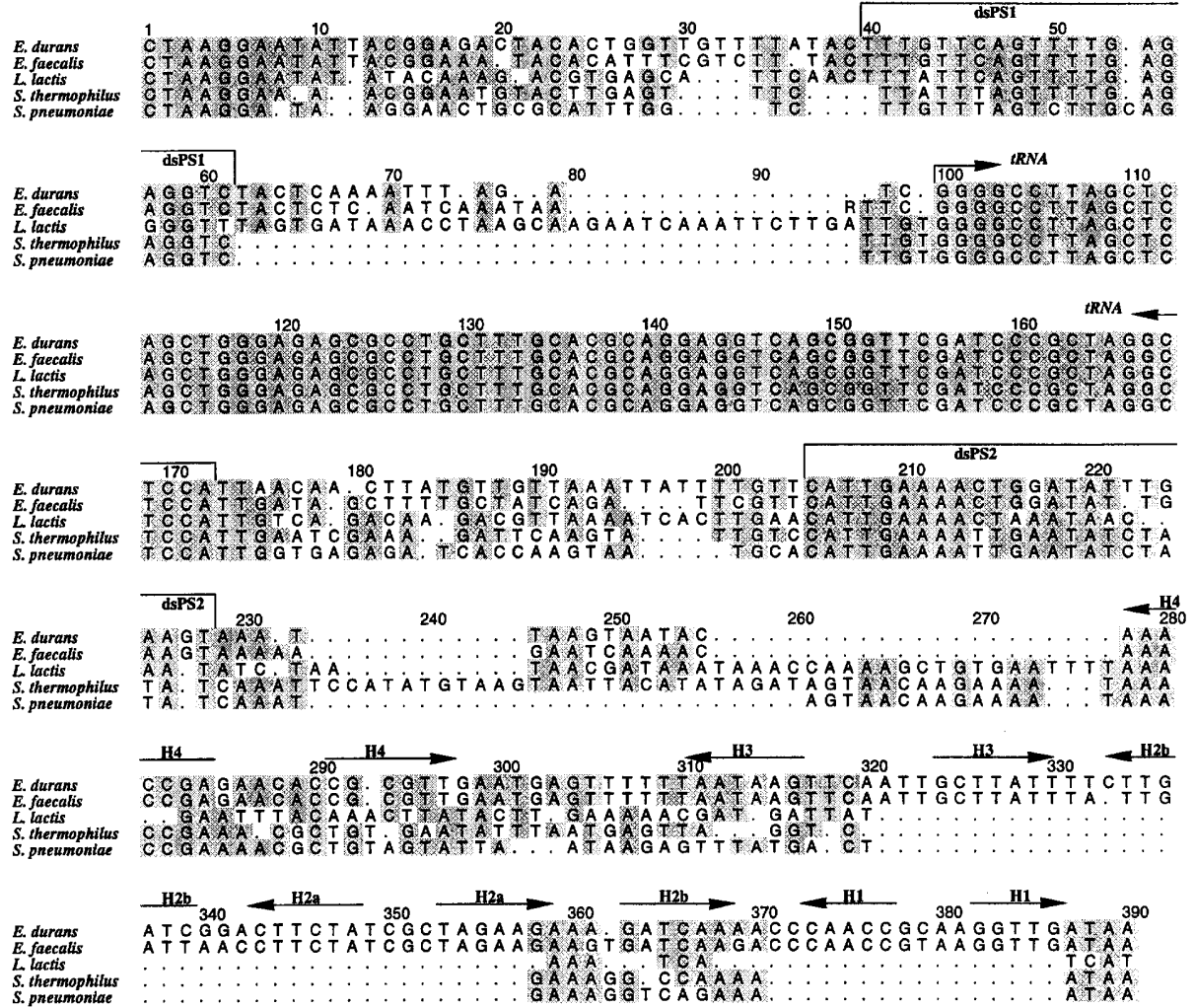

(b)
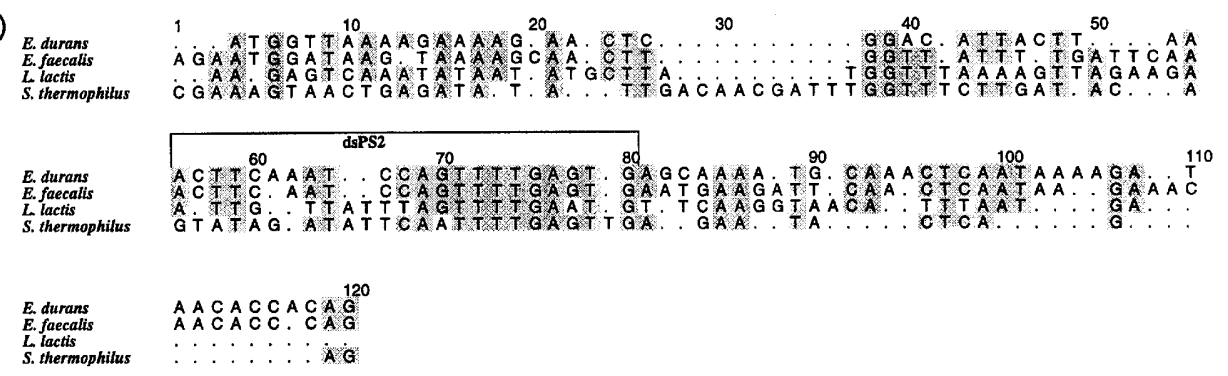

Fig. 5. Nucleotide sequence conservation of streptococcal spacer regions 1 and 2 . Multiple sequence alignment of the spacer regions 1 (a) and 2 (b) from one species of the 'E. faecium group' ( $E$. durans, this paper), $E$. faecalis (this paper and Hall, 1994), L. lactis (Chiaruttini \& Milet, 1993), S. thermophilus (Nour et al., 1995) were made with the CLUSTAL W program. Similarities are represented by grey shading as in Fig. 2 . In (a) the sequence of the $S$. pneumoniae spacer region 1 (Bacot \& Reeves, 1991) is included in the comparison. The sequences involved in the putative dsPS1 and dsPS2 stems are overlined. The tRNA ${ }^{A l a}$ and stem-loop structures $\mathrm{H} 1, \mathrm{H} 2, \mathrm{H} 3$ and $\mathrm{H} 4$ from enterococci (Fig. 4) are indicated.

genes (Ala, Glu and Leu) do not encode the $3^{\prime}$-terminal CCA (Green \& Vold, 1983), and tRNA genes without the $3^{\prime}$-terminal CCA were also found in a pentameric and in a hexameric tRNA gene cluster downstream of the 5S rRNA gene in Lactobacillus delbrueckeii subsp. bulgaricus (Pittet \& Hottinger, 1989), and in the distal $r r n$ spacer of L. lactis (Chiaruttini \& Milet, 1993) and of S. thermophilus (Guédon et al., 1992). Due to the redundancy of the CCA sequence at the $3^{\prime}$-end of the active $t R \mathrm{RA}^{\mathrm{Ala}}$, a CCA sequence is present at the $3^{\prime}$-end of the precursor tRNA in spacer region 1 and it may ensure maturation by RNase P. Maturation of tRNA ${ }^{A l a}$ from the pre-rRNA transcript by $R$ Nase $P$ probably depends primarily upon the tRNA ${ }^{\text {Ala }}$ structure, since the sequences that border the tRNA ${ }^{\text {Ala }}$ gene are highly variable among streptococci (Fig. 5a). Only a $5^{\prime}$ UUA/G-3' sequence on the $3^{\prime}$-side of the tRNA ${ }^{\mathrm{Ala}}$ is conserved.

Our comparative analysis of LS1 and SS1 showed pairs of palindromic and/or directly inverted sequences that may be related to the genetic events responsible for the presence of the two forms of spacer region 1 (Fig. 2). A $5^{\prime}$-CTCAA- $3^{\prime}$ sequence that always precedes the area of sequence divergence probably plays an important role in the process. Based on the identity of the nucleotide sequences of the SS1 and LS1 spacer region in a given strain, except for the substitution area, an homogenization of the nucleotide sequences from the SS1 and 
LS1 spacer regions by frequent DNA recombination events may be postulated.

Another example of the plasticity of the enterococcal spacer region 1 is the insertion of a $115 \mathrm{nt}$ stem-loop structure in the E. faecium spacer region 1 and of a $107 \mathrm{nt}$ stem-loop structure in one of the long versions of the E. hirae spacer region 1 (Fig. 4a). As the inserted sequence folds into a long stem-loop structure, the insertion does not alter the overall structure formed by spacer regions 1 and 2 (Fig. 4a). One open question is whether insertion of such stem-loop structures is an ancient or a recent event. Our observation of the $115 \mathrm{bp}$ insertion, in two E. faecium strains with very different origins, which has two sequence differences in spacer region 2 and four in spacer region 1, favours the hypothesis of an ancient event. However, according to the secondary structure they form and their occurrence in only one type of E. hirae rrn operons, the observed insertions share some common features with the mobile intervening sequences found in the $23 \mathrm{~S}$ rRNA coding sequence of some Gram-negative bacteria (reviewed by Konkel et al., 1994). A larger collection of E. faecium and E. hirae species will have to be studied to get a more definite answer. The high similarities of $E$. hirae and $E$. faecium spacer regions 1 and 2 (Table 2) and the presence of insertions suggest a close phylogenetic relationship between these two species.

Based on their poor nucleotide sequence conservation in streptococci, the spacer regions 1 and 2 are suitable targets for the design of genus-specific oligonucleotide probes. For instance, the sequence from positions 320 to 357 and from positions 371 to 386 (referring to the alignment in Fig. 5a) that display no significant homologies with the spacer regions 1 of lactococci and Streptococcus sensu stricto may be used as targets for the design of enterococci-specific DNA probes. If the inserts in E. faecium and E. hirae prove to be general, their sequences may be good targets for the design of species-specific DNA probes, and based on the alignment in Figs 2 and 3, several sequences from the spacer regions 1 and 2 could be tested as targets for the design of species-specific oligonucleotide probes. Another alternative for species identification may be PCR typing, since patterns of DNA fragments amplified with primer combination 1 differ markedly among species.

\section{ACKNOWLEDGEMENTS}

A.N. was a fellow of the Franco-Algerian Cooperation Organization. This work was supported by the French Centre National de la Recherche Scientifique and the French Ministère de la Recherche et de l'Enseignement supérieur. G. Mulliert is strongly thanked for his help in sequence alignment, Professor G. Lefebre is thanked for providing us with the $E$. faecium strain LMA631 and for helpful discussion. K. Harthmuth is thanked for his help in the establishment of phylogenetic trees and for reading this manuscript. J. Bayeul and $\mathrm{E}$. Habermacher are thanked for their technical assistance.

\section{REFERENCES}

Apirion, D. \& Miczak, A. (1993). RNA processing in prokaryotic cells. BioEssays 15, 113-120.

Bacot, C. M. \& Reeves, R. H. (1991). Novel tRNA gene organization in the 16S-23S intergenic spacer of the Streptococcus pneumoniae rRNA gene cluster. J Bacteriol 173, 4234 4236.

Barry, T., Colleran, G., Glennon, M., Dunican, L. K. \& Gannon, F. (1991). The $16 \mathrm{~s} / 23 \mathrm{~s}$ ribosomal spacer region as a target for DNA probes to identify eubacteria. PCR Methods Appl 1, 51-56.

Beimfohr, C., Krause, A., Amann, R., Ludwig, W. \& Schleifer, K. H. (1993). In situ identification of lactococci, enterococci and streptococci. Syst Appl Microbiol 16, 450-456.

Berg, K. L., Squires, C. \& Squires, C. L. (1989). Ribosomal RNA operon anti-termination-function of leader and spacer region boxB-boxA sequences and their conservation in diverse microorganisms. J Mol Biol 209, 345-358.

Betzl, D., Ludwig, W. \& Schleifer, K. H. (1990). Identification of lactococci and enterococci by colony hybridization with $23 \mathrm{~S}$ rRNA-targeted oligonucleotide probes. Appl Environ Microbiol 56, 2927-2929.

Borton, G. J. (1993). Alscript : a tool to format multiple sequence alignments. Protein Eng 6, 37-40.

Branlant, C., Krol, A., Machat, M. A., Pouet, J. \& Ebel, J. P. (1981). Primary and secondary structures of Escherichia coli MRE 600 ribosomal RNA. Comparison with models of secondary structures for maize chloroplast $23 \mathrm{~S}$ rRNA and for large portions of mouse and human $16 \mathrm{~S}$ mitochondrial rRNAs. Nucleic Acids Res 9, 4303-4324.

Chiaruttini, C. \& Milet, M. (1993). Gene organization, primary structure and RNA processing analysis of a ribosomal RNA operon in Lactococcus lactis. J Mol Biol 230, 57-76.

De Man, J. C., Rogosa, M. \& Sharpe, M. E. (1960). A medium for the cultivation of lactobacilli. J Appl Bacteriol 23, 130-135.

Devriese, L. A., Pot, B. \& Collins, M. D. (1993). Phenotypic identification of the genus Enterococcus and differentiation of phylogenetically distinct enterococcal species and species groups. $J$ Appl Bacteriol 75, 399-408.

van der Giessen, J. W. B., Haring, R. M. \& van der Zeijst, B. A. M. (1994). Comparison of the $23 S$ ribosomal RNA genes and the spacer region between the $16 \mathrm{~S}$ and $23 \mathrm{~S}$ rRNA genes of the closely related Mycobacterium avium and Mycobacterium paratuberculosis and the fast-growing Mycobacterium phlei. Microbiology 140, 1103-1108.

Green, C. J. \& Vold, B. S. (1983). Sequence analysis of cluster of twenty-one tRNA genes in Bacillus subtilis. Nucleic Acids Res 11, 5763-5774.

Guédon, G., Pébay, M., Colmin, C., Simonet, J. M. \& Decaris, B. (1992). The 23S-5S spacer of two rRNA loci of Streptococcus salivarius subsp. thermophilus include a promoter. Biochimie 74 , 585-588.

Gurtler, V. \& Stanisich, V. A. (1996). New approaches to typing and identification of bacteria using the $16 \mathrm{~S}-23 \mathrm{~S}$ rDNA spacer region. Microbiology 142, 3-16.

Hall, L. M. C. (1994). Are point mutations or DNA rearrangements responsible for the restriction fragment length polymorphisms that are used to type bacteria? Microbiology 140, 197-204.

Hall, L. M. C., Duke, B., Guiney, M. \& Williams, R. (1992). Typing of Enterococcus species by DNA restriction fragment analysis. $J$ Clin Microbiol 30, 915-919.

Harasawa, R., Uemori, T., Asada, K., Kato, I. \& Shiragami, N. 
(1992). 'boxA'-like sequence between the $16 \mathrm{~S} / 23 \mathrm{~S}$ spacer in rRNA operon of mycoplasmas. FEBS Lett 297, 209-211.

Hardie, J. M. (1986). Genus Streptococcus Rosenbach 1884. In Bergey's Manual of Systematic Bacteriology, vol. 2, pp. 1043-1047. Edited by P. H. A. Sneath, N. S. Mair, M. E. Sharpe \& J. G Holt. Baltimore: Williams \& Wilkins.

Hill, C., Miller, L. A. \& Klaenhamer, T.R. (1990). Cloning, expression, and sequence determination of a bacteriophage fragment encoding bacteriophage resistance in Lactococcus lactis. J Bacteriol 172, 6419-6426.

Jensen, M. A., Webster, J. A. \& Straus, N. (1993). Rapid identification of bacteria on the basis of polymerase chain reactionamplified ribosomal DNA spacer polymorphisms. Appl Environ Microbiol 59, 945-952.

Ji, Y., Kempsell, K. E., Colston, M. J. \& Cox, R. A. (1994). Nucleotide sequences of the spacer-1, spacer-2 and trailer regions of the $r r n$ operons and secondary structures of precursor $23 \mathrm{~S}$ rRNAs and precursor $5 \mathrm{~S}$ rRNAs of slow-growing mycobacteria. Microbiology 140, 1763-1773.

Kilpper-Bălz, R., Fischer, G. \& Schleifer, K. H. (1982). Nucleic acid hybridization of group $\mathrm{N}$ and group $\mathrm{D}$ streptococci. Curr Microbiol 7, 245-250.

Konkel, M. E., Marconi, R. T., Head, D. J. \& Cieplak, W. (1994). Identification and characterization of an intervening sequence within the $23 \mathrm{~S}$ ribosomal RNA genes of Campylobacter jejuni. Mol Microbiol 14, 235-241.

Le Bourgeois, P., Lautier, M., Van Den Berghe, L., Gasson, M. J. \& Ritzenthaler, P. (1995). Physical and genetic map of the Lactococcus lactis subsp. cremoris MG1363 chromosome: comparison with that of Lactococcus lactis subsp. lactis IL 1403 reveals a large genome inversion. J Bacteriol 177, 2840-2850.

Loughney, K., Lund, E. \& Dahlberg, J. E. (1982). tRNA genes are found between the $16 \mathrm{~S}$ and the $23 \mathrm{~S}$ rRNA genes in Bacillus subtilis. Nucleic Acids Res 10, 1607-1624.

Ludwig, W., Seewaldt, E., Kilpper-Bălz, R., Schleifer, K. H., Magrum, L., Woese, C. R., Fox, G. E. \& Stackebrandt, E. (1985). The phylogenetic position of Streptococcus and Enterococcus. J Gen Microbiol 131, 543-551.

Ludwig, W., Kirchhof, G., Klugbauer, N. \& 13 other authors (1992). Complete $23 S$ ribosomal RNA sequences of Gram-positive bacteria with a low DNA G + C content. Syst Appl Microbiol 15, 487-501.

Mundt, J. O. (1986). Enterococci. In Bergey's Manual of Systematic Bacteriology, vol. 2, pp. 1063-1065. Edited by P. H. A. Sneath, N. S. Mair, M. E. Sharpe \& J. G. Holt. Baltimore: Williams \& Wilkins.

Nour, M., Naïmi, A., Beck, G. \& Branlant, C. (1995). 16S-23S and 23S-5S intergenic spacer regions of Streptococcus thermophilus and Streptococcus salivarius, primary and secondary structure. Curr Microbiol 31, 270-278.

Ogasawara, N., Moriya, S. \& Yoshikawa, H. (1983). Structure and organization of $\mathrm{rRNA}$ operons in the region of the replication origin of the Bacillus subtilis chromosome. Nucleic Acids Res 18, $6301-6318$.

Pébay, M., Colmin, C., Guédon, G., De Gaspéri, C., Decaris, B. \& Simonet, J. M. (1992). Detection of intraspecific DNA polymorphism in Streptococcus salivarius subsp. thermophilus by a homologous rDNA probe. Res Microbiol 143, 37-46.

Pernodet, J. L., Boccard, F., Alegre, M. T., Gagnat, J. \& Guérineau, M. (1989). Organization and nucleotide sequence of a ribosomal RNA gene cluster from Streptomyces ambofaciens. Gene 79, $33-46$.

Pittet, A. C. \& Hottinger, H. (1989). Sequence of a hexameric tRNA gene cluster associated with rRNA genes in Lactobacillus bulgaricus. Nucleic Acids Res 17, 4873.

Potter, S., Durovic, P. \& Dennis, P. P. (1995). Ribosomal RNA precursor processing by a eukaryotic U3 small nucleolar RNAlike molecule in an archaeon. Nature 268, 1056-1060.

Sambrook, J., Fritsch, E. F. \& Maniatis, T. (1989). Molecular Cloning: a Laboratory Manual, 2nd edn. Cold Spring Harbor, NY: Cold Spring Harbor Laboratory.

Schleifer, K. H. \& Kilpper-Bălz, R. (1987). Molecular and chemotaxonomic approaches to the classification of streptococci, enterococci and lactococci: a review. Syst Appl Microbiol 10, 1-19.

Sechi, L. A. \& Daneo-Moore, L. (1993). Characterization of intergenic spacers in two rrn operons of Enterococcus hirae ATCC 9790. J Bacteriol 175, 3213-3219.

Tabor, S. \& Richardson, C. C. (1987). DNA sequence analysis with a modified bacteriophage T7 DNA polymerase. Proc Natl Acad Sci USA 84, 4767-4771.

Thompson, J. D., Higgins, D. G. \& Gibson, T. J. (1994). ClusTal. w : improving the sensitivity of progressive multiple sequence alignment through sequence weighting, position-specific gap penalties and weight matrix choice. Nucleic Acids Res 22, 4673-4680.

Whiley, R. A., Duke, B., Hardie, J. M. \& Hall, L. M. C. (1995). Heterogeneity among 16S-23S rRNA intergenic species within the 'Streptococcus milleri group'. Microbiology 141, 1461-1467.

Williams, A. M., Rodrigues, U. M. \& Collins, M. D. (1991). Intrageneric relationships of enterococci as determined by reverse transcriptase sequencing of small-subunit rRNA. Res Microbiol $142,67-74$.

Received 24 April 1996; revised 24 October 1996; accepted 19 November 1996. 